\title{
Halt Optimization Strategy for a Space Manipulator with a Joint- Locked Failure
}

\author{
Gang Chen $(\mathbb{D}$, Lanpu Li $\mathbb{D}$, Yingzhuo Fu, Bonan Yuan, and Junting Fei \\ School of Automation, Beijing University of Posts and Telecommunications, Beijing 100876, China \\ Correspondence should be addressed to Gang Chen; buptcg@163.com
}

Received 20 May 2020; Revised 8 August 2020; Accepted 24 August 2020; Published 7 September 2020

Academic Editor: Antonio Concilio

Copyright (C) 2020 Gang Chen et al. This is an open access article distributed under the Creative Commons Attribution License, which permits unrestricted use, distribution, and reproduction in any medium, provided the original work is properly cited.

\begin{abstract}
Aiming at a space manipulator with a joint-locked failure, a halt optimization strategy is proposed in this paper. Firstly, a halt configuration optimization model (HCOM) is constructed, to select an optimal configuration where the kinematic ability of the manipulator is the best. Secondly, considering the constraint of joint running parameters and the disturbance torque of the base, we construct and solve the halt motion optimization model (HMOM), which can achieve a steady halt and ensure the safety of the manipulator. The correctness and effectiveness of the proposed strategy in this paper are verified with a 7-DOF space manipulator. This strategy firstly puts forward the idea of halt configuration optimization and realizes the minimum global disturbance torque of the base in the halt process.
\end{abstract}

\section{Introduction}

As a high-end space equipment with large span, high flexibility, and strong operation capability, space manipulators have been widely used in the field of space exploration [1-5]. However, due to the complex joint structure and hostile working environment (high-temperature difference, high radiation, etc.), a joint-locked failure is prone to occur [6-9]. The faulty joint is locked at the failure moment and loses the ability to move. At this time, if the manipulator executes the original task without halt, its running parameters (joint velocity, acceleration, torque, etc.) will jump at the failure moment [10-12], bringing a deformation or more damage to the manipulator [13-15]. Therefore, to ensure a safe and stable operation, the faulty manipulator needs to be halted after the joint-locked failure occurs.

The halt of the space manipulator with a joint-locked failure aims at designing a subsequent motion to halt the manipulator in a certain configuration. Aiming at the halt of a faulty manipulator, some scholars have carried out relevant researches. Kamata et al. [16] achieved an emergency stop of the faulty manipulator by utilizing the joint braking based on the joint limit. Dupac and Noroozi [17] proposed a halt strategy by limiting the impact of the end of the manipulator, realizing the halt of the manipulator. To avoid the joint overload, Nozawa et al. [18] achieved the halt of the manipulator by limiting the joint running parameters. In the above study, considering the parameter constraints of the manipulator, the corresponding motion planning strategy is constructed to realize the shutdown of the manipulator. But the above halt methods only consider the constraints of joint parameters, ignoring the characteristic that the running parameters of the manipulator are likely to mutate. That is to say, these halt methods belong to an emergency stop, which will seriously affect the safety of the manipulator in the halt process [19, 20]. Therefore, within relevant constraint conditions, the running parameters need to be optimized during the halt process for the faulty manipulator.

Aiming at the halt optimization of a manipulator with a joint-locked failure, $\mathrm{Xu}$ et al. [21] used a sequence quadratic program (SQP) to obtain the halt trajectory with optimal torque by considering the torque limitation. Gao et al. [22] optimized the joint velocity based on the linear and cosine curve with the joint torque as the constraint condition. To optimize the energy consumption in the halt process, Dalla and Pathak [23] proposed a halt optimization method by limiting the joint velocity, which achieved 
a halt with the minimum power consumption. Although the above researches realized the halt optimization of the manipulator with a joint-locked failure, they do not consider the halt configuration, which means that the halt configuration of the manipulator is arbitrary. The space manipulator has different kinematic ability under different configurations. Considering that on-orbit operation tasks have high requirements on the kinematic ability of the space manipulator $[24,25]$, if the manipulator is stopped at any configuration (such as the singular configuration), its kinematic ability in the anchor configuration may not meet task requirements, which seriously restricts the reliable execution of the subsequent operation task. Therefore, the manipulator should be stopped in an optimal configuration according to task requirements, so that it can reliably perform the subsequent operation task with the best kinematic ability.

In addition, the existing researches on the halt optimization of the manipulator are only aimed at the ground manipulator. But for the space manipulator, its base usually floats freely $[26,27]$. If the above methods for ground manipulators are directly applied to the space manipulator, it will generate a disturbance torque on the base in the halt process because of the motion coupling between the manipulator and the base [28], and the attitude deflection of the base will be caused. However, if the attitude deflection of the base is too large, the spacecraft's communication quality and energy acquisition efficiency will be reduced, and even the manipulator will be out of control, seriously threatening the safety of the space manipulator [29]. For this reason, to solve the problem of the halt optimization of the manipulator with a joint-locked failure, the disturbance torque of base should be limited within a small range.

In summary, this paper proposes a halt optimization strategy for the space manipulator with a joint-locked failure, which is made up of two parts: (1) A comprehensive characterization of the kinematic ability, covering the manipulator itself and task requirements, is obtained. Then, a halt configuration optimization model (HCOM) is constructed with the best kinematic ability under the halt configuration as the objective. By solving the HCOM, the optimal halt configuration is obtained. (2) The halt motion is planned by using a six-order polynomial interpolation method which contains an optimization coefficient. Considering the motion coupling between the base and the manipulator, a halt motion optimization model (HMOM) is constructed with the minimum disturbance torque of the base as the objective and joint parameters as the constraint. With the HMOM solved, the halt motion optimization is achieved which can realize the steady halt and ensure the safety of the manipulator in the halt process.

Aiming at the space manipulator with a joint-locked failure, the innovation of this paper mainly is shown as follows

(1) The halt configuration is optimized, ensuring that the manipulator in the halt configuration owns the best kinematic ability, and the kinematic ability in the optimal halt configure is $236.8 \%$ higher than that in the suboptimal halt configuration
(2) Each unilateral kinematic ability index is analyzed quantitatively, making the comprehensive representation of the kinematic ability more accurate, which can guarantee the execution of subsequent task

(3) The disturbance torque of the base is optimized in the halt process, and its global disturbance torque is reduced by $30 \%$ at least after the optimization

The rest of this paper is organized as follows: In Section 2, we analyze the halt optimization problem and describe it as a halt configuration optimization problem (HCOP) and a halt motion optimization problem (HMOP). In Section 3, aiming at the HCOP, a HCOM is constructed and solved to obtain the optimal halt configuration. In Section 4, aiming at the HMOP, a HMOM is constructed and solved to achieve the halt optimization. In Section 5, the simulation is carried on to verify the effectiveness of the proposed strategy. In Section 6 , the conclusions are summarized.

\section{Materials and Methods}

2.1. Problem Description. In this section, a halt optimization problem of a space manipulator with a joint-locked failure is analyzed. The halt optimization problem is described as the HCOP and the HMOP by considering task requirements and the characteristic of the base.

To express easily, the symbols involved in this section are annotated in Table 1. And the halt optimization problem can be described as considering the constraint lim and the optimization objective $\boldsymbol{V a r}$, take $\boldsymbol{S}_{0}$ as input, and optimize the halt process to make the manipulator come to $S_{f}$.

Considering the high requirements of the subsequent operation tasks, it is necessary to make the kinematic ability of the manipulator under the halt configuration as high as possible. In other words, the halt configuration should be optimized with the best kinematic ability as the objective. Let the kinematic ability under the configuration $\boldsymbol{q}$ be $\mathbf{C P}$ $(\boldsymbol{q})$, and the HCOP can be described as

$$
\begin{aligned}
& \text { find } \boldsymbol{q}_{\text {best }}, \\
& \max \boldsymbol{C P}\left(\boldsymbol{q}_{\text {best }}\right),
\end{aligned}
$$

where $\boldsymbol{q}_{\text {best }}$ is the optimal halt configuration.

Considering the motion coupling between the manipulator and the base, we take the minimum base disturbance torque as the objective and limit the joint parameters (angle, velocity, and acceleration) within a range. On the basis, the manipulator is stably halted to $\boldsymbol{q}_{\text {best }}$. The HMOP can be described as

$$
\begin{array}{lc}
\min & \boldsymbol{\tau}_{b} \\
\text { s.t. } & \lim \\
& \boldsymbol{q}_{f}=\boldsymbol{q}_{\text {best }} .
\end{array}
$$

At this point, the halt optimization problem is transformed into the HCOP and the HMOP, which is shown in Figure 1. 
TABLE 1: Symbols annotations of the halt.

\begin{tabular}{|c|c|}
\hline Symbols & Annotations \\
\hline$t_{0}$ & The initial time of the halt \\
\hline$t_{f}$ & The end time of the halt \\
\hline$t$ & The time during the halt \\
\hline $\boldsymbol{q}_{t}$ & The configuration when the time is $t$ \\
\hline$\dot{\boldsymbol{q}}_{t}$ & The joint velocity when the time is $t$ \\
\hline$\ddot{\boldsymbol{q}}_{t}$ & The joint acceleration when the time is $t$ \\
\hline$\tau_{b}$ & The disturbance torque of the base in the halt process \\
\hline$S_{0}$ & The initial state of the halt, $\boldsymbol{S}_{0}=\left[\boldsymbol{q}_{0}, \dot{\boldsymbol{q}}_{0}, \ddot{\boldsymbol{q}}_{0} \cdots\right]$ \\
\hline$S_{f}$ & The end state of the halt, $\boldsymbol{S}_{f}=\left[\boldsymbol{q}_{f}, \dot{\boldsymbol{q}}_{f}, \ddot{\boldsymbol{q}}_{f} \cdots\right]$ and $\dot{\boldsymbol{q}}_{f}=\mathbf{0}, \ddot{\boldsymbol{q}}_{f}=\mathbf{0}$ \\
\hline Var & The parameters of the manipulator in the halt process, $\operatorname{Var}=\left\{\boldsymbol{q}_{t}, \dot{\boldsymbol{q}}_{t}, \ddot{\boldsymbol{q}}_{t}, \boldsymbol{\tau}_{\mathrm{b}} \cdots\right\}$ \\
\hline $\lim$ & The constraint of the halt, lim $=\left\{\left[\boldsymbol{q}_{\min }, \boldsymbol{q}_{\max }\right],\left[\dot{\boldsymbol{q}}_{\min }, \dot{\boldsymbol{q}}_{\max }\right],\left[\ddot{\boldsymbol{q}}_{\min }, \ddot{\boldsymbol{q}}_{\max }\right],\left[\boldsymbol{\tau}_{\min }, \boldsymbol{\tau}_{\max }\right] \cdots\right\}$ \\
\hline
\end{tabular}

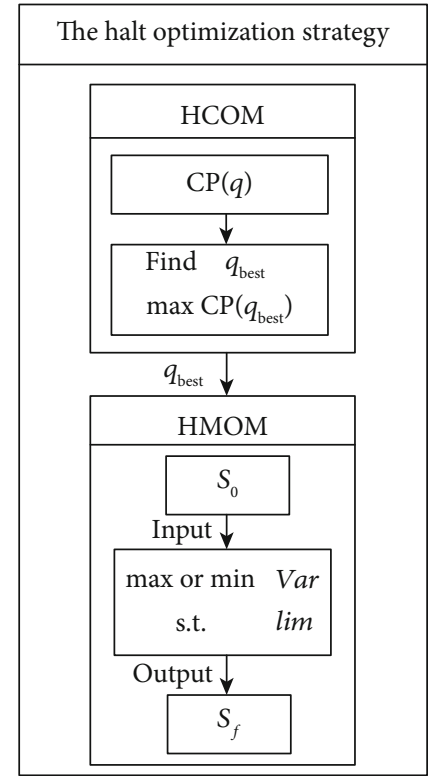

FIgURE 1: The description of the halt optimization problem.

\section{The Halt Configuration Optimization}

The halt configuration optimization of the space manipulator with a joint-locked failure is to select the optimal configuration with the best kinematic ability, which can ensure that the manipulator under the halt configuration can meet task requirements. In this section, aiming at the $\mathrm{HCOP}$, the kinematic ability of the manipulator is comprehensively characterized, and then the HCOM is constructed and solved based on the Monte Carlo method to obtain the optimal halt configuration.

3.1. Comprehensive Characterization of the Kinematic Ability. The representation of the kinematic ability of the space manipulator depends on its kinematic model. However, the original kinematic model of the space manipulator



FIgURE 2: The kinematic model of $n$-DOF space manipulator without a joint-locked failure.

is no longer applicable due to the locked joint. For this reason, this section firstly reconstructs the kinematic model and then analyzes the unilateral kinematic ability indexes. Finally, the kinematic ability of the manipulator is comprehensively characterized based on the traditional entropy method and analytic hierarchy process (AHP) method.

3.1.1. The Reconstruction of the Kinematic Model. For the nDOF space manipulator without a joint-locked failure, its kinematic model is shown in Figure 2. To express easily, the symbols involved in this section are annotated in Table 2.

Assuming that the $J_{l}$ is locked, and the link $L_{l-1}$ and link $L_{l}$ can be connected as a new $\operatorname{link} \tilde{L}_{l-1}$. Specifying the coordinate system of the link and joint after $J_{l}$ is locked as follows: If the location of the joint or link is in front of the $J_{l}$, it is consistent with the kinematic model without a joint-locked failure; otherwise, add " " above the symbols of the kinematic model without a joint-locked failure, and the new number of the joint or link is subtracted 1 from the original basis. 
TABLE 2: Symbols annotations of $n$-DOF space manipulator without a joint-locked failure.

\begin{tabular}{|c|c|}
\hline Symbols & Annotations \\
\hline$\Sigma_{I}$ & Inertial coordinate frame \\
\hline$\Sigma_{b}$ & Base coordinate system \\
\hline$\Sigma_{e}$ & End-effector coordinate system \\
\hline$\Sigma_{i}$ & The coordinate system of the $i$-th link $(i=1,2, \cdots, n)$ \\
\hline$C_{i}$ & Centroid of the $i$-th link $(i=1,2, \cdots, n)$ \\
\hline$J_{i}$ & $\begin{array}{l}\text { The joint connected }(i-1) \text {-th link and } i \text {-th link } \\
\qquad(i=1,2, \cdots, n)\end{array}$ \\
\hline $\mathbf{l}_{i}$ & The vector from $J_{i}$ to $J_{i+1}$, and $\mathbf{l}_{i}=\mathbf{a}_{i}+\mathbf{b}_{i}$ \\
\hline $\mathbf{a}_{i}$ & The vector from $J_{i}$ to $C_{i}(i=1,2, \cdots, n)$ \\
\hline $\mathbf{b}_{i}$ & The vector from $C_{i}$ to $J_{i+1}(i=1,2, \cdots, n-1)$ \\
\hline $\mathbf{b}_{n-1}$ & The vector from $C_{n-1}$ to the end-effector \\
\hline${ }^{I} \mathbf{z}_{i}$ & The unit vector in the axis direction in $\Sigma_{i}$ \\
\hline$m_{i}$ & The mass of the $i$-th link $(i=1,2, \cdots, n)$ \\
\hline$m_{b}$ & The mass of the base \\
\hline$M$ & The total mass of the system \\
\hline $\mathbf{I}_{i}$ & The inertia tensor of $i$-th link $(i=1,2, \cdots, n)$ \\
\hline $\mathbf{I}_{b}$ & The inertia tensor of base \\
\hline$\theta_{i}$ & The joint angle of $i$-th joint $(i=1,2, \cdots, n)$ \\
\hline $\mathbf{q}_{\theta}$ & $\begin{array}{l}\text { The position vector of joint angle in joint space, } \\
\qquad \boldsymbol{q}_{\theta}=\left[\theta_{1}, \cdots, \theta_{n}\right]^{\mathrm{T}} \in \mathfrak{R}^{n \times 1}\end{array}$ \\
\hline${ }^{I} \mathbf{r}_{b}$ & The position vector of centroid of base in inertial frame \\
\hline${ }^{I} \mathbf{r}_{i}$ & $\begin{array}{l}\text { The position vector of centroid of } i \text {-th in inertial frame } \\
\qquad(i=1,2, \cdots, n)\end{array}$ \\
\hline${ }^{I} \mathbf{r}_{\mathrm{e}}$ & The position vector of the end-effector \\
\hline${ }^{I} \mathbf{p}_{i}$ & The position vector of $J_{i}(i=1,2, \cdots, n)$ \\
\hline $\mathbf{q}_{b}$ & The pose vector of the base, $\mathbf{q}_{b}=\left[\boldsymbol{r}_{b}, \boldsymbol{\phi}_{b}\right]$ \\
\hline$\phi_{b}$ & The orientation vector of the base \\
\hline$\dot{\mathbf{q}}_{b}$ & The velocity of the base \\
\hline$x_{e}$ & The pose vector of the end-effector, $\boldsymbol{x}_{e}=\left[\boldsymbol{r}_{e}, \boldsymbol{\phi}_{e}\right]$ \\
\hline$\phi_{e}$ & The orientation vector of the end-effector \\
\hline $\mathbf{v}_{b}$ & The velocity of the centroid of base \\
\hline $\mathbf{v}_{i}$ & The velocity of the centroid of $i$-th link $(i=1,2, \cdots, n)$ \\
\hline $\mathbf{v}_{e}$ & The velocity of the end-effector \\
\hline $\boldsymbol{\omega}_{b}$ & The angular velocity of the base \\
\hline$\omega_{i}$ & The angular velocity of the $i$-th link \\
\hline $\boldsymbol{\omega}_{e}$ & The angular velocity of the end-effector \\
\hline
\end{tabular}

The dynamics parameters of $\tilde{L}_{l-1}$ can be obtained according to the following equation

(1) The mass of $\tilde{L}_{l-1}: \tilde{\boldsymbol{m}}_{\boldsymbol{l - 1}}=\boldsymbol{m}_{\boldsymbol{l}}+\boldsymbol{m}_{\boldsymbol{l - 1}}$

(2) The centroid of $\tilde{L}_{l-1}:{ }^{I} \tilde{\mathbf{r}}_{\boldsymbol{l}-1}=\left(\boldsymbol{m}_{\boldsymbol{l - 1}}{ }^{I} \mathbf{r}_{\boldsymbol{l}-1}+\boldsymbol{m}_{\boldsymbol{l}}{ }^{I} \mathbf{r}_{\boldsymbol{l}}\right) /($ $\left.m_{l-1}+m_{l}\right)$

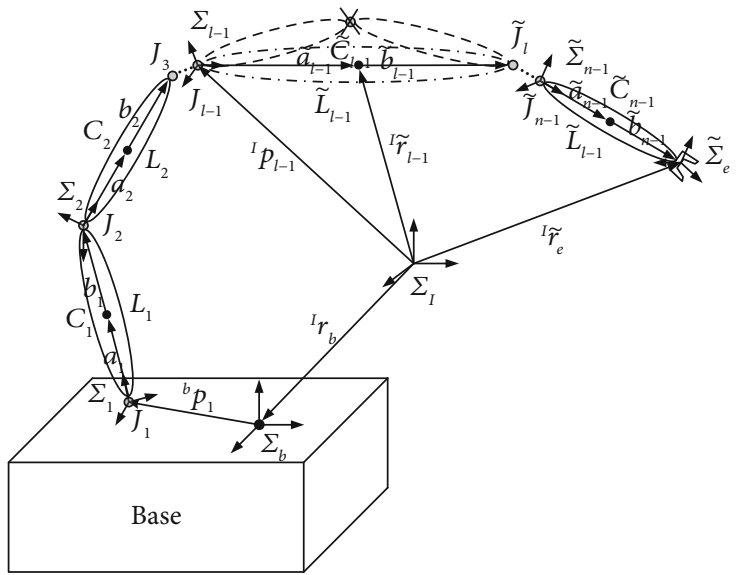

FIgURE 3: The reconstructed kinematic model of $n$-DOF space manipulator with a joint-locked failure.

(3) The inertia tensor of $\tilde{L}_{l-1}: \tilde{\mathbf{I}}_{\boldsymbol{l}-1}=\mathbf{I}_{\boldsymbol{l}-1}+\boldsymbol{m}_{\boldsymbol{l - 1}}\left(\left({ }^{I} \mathbf{r}_{\boldsymbol{l - 1}}\right)^{T}\right.$ $\left.{ }^{I} \mathbf{r}_{l-1} \mathbf{E}_{3}-{ }^{I} \mathbf{r}_{l-1}\left({ }^{I} \mathbf{r}_{l-1}\right)^{T}\right)$, where $\mathbf{E}_{3} \in \Re^{3 \times 3}$ represents the third order identity matrix

Thus, the reconstruction of the kinematic model is shown in Figure 3.

The velocity of the end-effector is represented as

$$
\dot{\tilde{\mathbf{x}}}_{e}=\tilde{\mathbf{J}}_{b} \dot{\tilde{\mathbf{q}}}_{b}+\tilde{\mathbf{J}}_{\theta} \dot{\tilde{\mathbf{q}}}_{\theta}
$$

where $\tilde{\mathbf{J}}_{b}$ represents the base Jacobian matrix; $\tilde{\mathbf{J}}_{\theta}$ represents the joint Jacobian matrix.

Since the base of the space manipulator is in a freefloating state and is not influenced by external forces, the momentum of the system is conserved. Assuming that the momentum zero in the initial state, the velocity relation between the base and the end-effector can be expressed as

$$
\dot{\tilde{\mathbf{q}}}_{b}=\tilde{\mathbf{J}}_{b \theta} \dot{\tilde{\mathbf{q}}}_{\theta}
$$

where $\tilde{\mathbf{J}}_{b \theta}$ represents the base-joint Jacobian matrix.

Substituting Equation (3) into Equation (4), we can obtain

$$
\dot{\tilde{\mathbf{x}}}_{e}=\left(\dot{\mathbf{J}}_{\theta}+\dot{\mathbf{J}}_{b} \tilde{\mathbf{J}}_{b \theta}\right) \dot{\tilde{\mathbf{q}}}_{\theta}=\tilde{\mathbf{J}}_{F} \dot{\tilde{\mathbf{q}}}_{\theta},
$$

where $\tilde{\mathbf{J}}_{F}$ represents the generalized Jacobian matrix.

3.1.2. The Analysis of the Unilateral Kinematic Ability. The kinematic ability of the space manipulator generally includes the minimum singular value, condition number, and manipulability, which are related to the singular value of the Jacobian matrix [30]. Next, the kinematic ability will be analyzed based on $\tilde{\mathbf{J}}_{F}$.

According to the singular value decomposition theory, $\tilde{\mathbf{J}}_{F}$ can be written as

$$
\tilde{\boldsymbol{J}}_{F}=\boldsymbol{U} \boldsymbol{\Sigma} \boldsymbol{V},
$$


TABLE 3: The unilateral kinematic ability of the space manipulator with a joint-locked failure.

\begin{tabular}{lccc}
\hline & \multicolumn{1}{c}{ Minimum singular value } & Condition number & Manipulability \\
\hline Expression & $s=\sigma_{m}$ & $k=\sigma_{1} / \sigma_{m}$ & $w=\sigma_{1} \sigma_{2} \cdots \sigma_{m}$ \\
Meaning & $\begin{array}{c}\text { The degree to which the manipulator } \\
\text { approaches the singular configuration }\end{array}$ & $\begin{array}{c}\text { The condition number reflects the } \\
\text { isotropy of the manipulator's } \\
\text { kinematic ability in all directions. }\end{array}$ & $\begin{array}{c}\text { The measure of the end-effector's } \\
\text { kinematic ability when manipulator moves } \\
\text { in different directions }\end{array}$ \\
$\begin{array}{l}\text { Value } \\
\text { range }\end{array}$ & $s \in[0,+\infty)$ & $k \in[1,+\infty)$ & $w \in[0,+\infty)$ \\
$\begin{array}{l}\text { Value } \\
\text { analysis }\end{array}$ & $\begin{array}{c}\text { The smaller the minimum singular value } \\
\text { is, the closer the configuration is to the } \\
\text { singular configuration. }\end{array}$ & $\begin{array}{c}\text { The closer the condition number is to } \\
1, \text { the higher the dexterity of the } \\
\text { manipulator is. }\end{array}$ & $\begin{array}{c}\text { The bigger degenerate manipulability is, } \\
\text { the greater kinematic ability of the end- } \\
\text { effector in all directions is. }\end{array}$
\end{tabular}

where $\mathbf{U} \in \mathfrak{R}^{m \times m}$ and $\mathbf{V} \in \mathfrak{R}^{(n-1) \times(n-1)}$ are orthogonal matrices; $\Sigma \in \mathfrak{R}^{m \times(n-1)}$ is diagonal and nonnegative real rectangular diagonal matrix, and $\Sigma$ can be written as

$$
\boldsymbol{\Sigma}=\left[\begin{array}{ccccc}
\sigma_{1} & 0 & \cdots & 0 & \mathbf{0} \\
0 & \sigma_{2} & \cdots & 0 & \mathbf{0} \\
\vdots & \vdots & \ddots & \vdots & \mathbf{0} \\
0 & 0 & \cdots & \sigma_{m} & \mathbf{0}
\end{array}\right],
$$

where $\sigma_{1}$ is the maximum singular value; $\sigma_{m}$ is the minimum singular value. When the space manipulator is in a singular configuration, $\sigma_{m}=0$.

According to the singular values obtained above, the unilateral kinematic ability of the space manipulator can be obtained, as shown in Table 3.

According to Table 3, the bigger minimum singular value and manipulability is, the better the kinematic ability is. And the smaller condition number is, the better the kinematic ability is. As a consequence, it is necessary to carry out a standardized treatment for the above unilateral kinematic ability.

The minimum singular value and the manipulability are standardized as

$$
\begin{aligned}
& \widehat{s}=\operatorname{standard}(s)=\frac{(s-a)}{(b-a)}, \\
& \widehat{w}=\operatorname{standard}(w)=\frac{(w-a)}{(b-a)},
\end{aligned}
$$

where standard $(x)=(x-a) /(b-a), x \in[a, b]$ represents the unilateral kinematic ability; $\widehat{s}$ represents the minimum singular value with standardization, and $\widehat{w}$ represents the manipulability with standardization.

Aiming at the condition number, it can be standardized as

$$
\widehat{k}=\frac{1}{k}
$$

where $\widehat{k}$ represents the condition number with standardization.

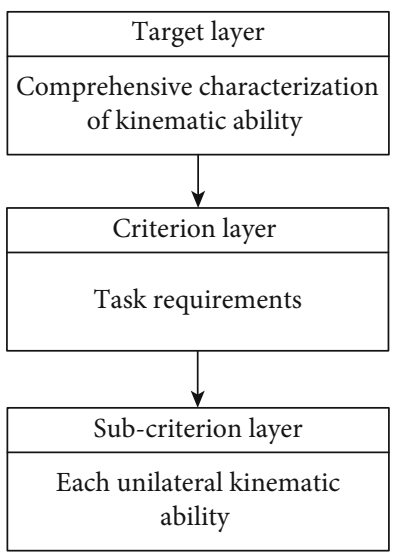

FIgURE 4: The hierarchical substructure model.

At this point, each unilateral kinematic ability is standardized, and then the kinematic ability will be comprehensively characterized.

3.1.3. The Comprehensive Characterization of the Kinematic Ability. The key to the comprehensive characterization of the kinematic ability is to determine the weight of each unilateral kinematic ability. The traditional entropy method ignores the qualitative factor that different tasks have different requirements on each unilateral kinematic ability. For example, assuming that a task needs the higher isotropy, the weight solved by the traditional entropy method only considers the unilateral kinematic ability and cannot reflect the higher isotropy, leading to an inaccuracy for the comprehensive characterization of kinematic ability. Considering that the AHP method can be used for the qualitative analysis [31], we choose the AHP method for the comprehensive characterization. Therefore, to cover the manipulator and requirements of the task, we combine and improve the AHP method and the traditional entropy method.

Firstly, we calculate the subjective weight by utilizing the AHP method.

(1) Construct the hierarchical substructure model. Considering that different task requires different kinematic ability, a hierarchical structure from top to bottom is constructed as Figure 4, including target layer (comprehensive characterization of 
TABLE 4: Scale definitions of 1-7 scale method.

\begin{tabular}{lc}
\hline Scale & Definition \\
\hline 1 & $i$ is just as important as $j$ \\
3 & $i$ is slightly more important than $j$ \\
5 & $i$ is more important than $j$ \\
7 & $i$ is even more important than $j$ \\
$2,4,6$ & The importance lies between adjacent scales \\
Reciprocal & If the ratio of $i$ and $j$ is $r_{i j}$, the ratio of $j$ and $i$ is $r_{j i}=$ \\
& $1 / r_{i j}$ \\
\hline
\end{tabular}

kinematic ability), criterion layer (task requirements), and subcriterion layer (each unilateral kinematic ability). The target layer is the highest layer in the hierarchy, and the criterion layer and subcriterion layer are used to evaluate the subjective weight

(2) Construct judgment matrix. To judge the influence of factors at the current level on that at the next level, 1-7 scale method as shown in Table 4 is used to define the importance degree of each element [32], and we construct the judgment matrix of Target-Criterion $\boldsymbol{R}=\left(r_{i j}\right)_{M \times N}$

Based on Table 4, the judgment matrix $\boldsymbol{R}=\left(r_{i j}\right)_{M \times N}$ can be obtained by comparing the importance of each kinematic ability.

(3) Do consistency test on the judgment matrix. By utilizing the maximum eigenvalue method, we can get the maximum eigenvalue of $\boldsymbol{R}=\left(r_{i j}\right)_{M \times N}$, and then the consistency test is used to judge whether the matrix is reasonable or not

Solve the consistency index CI

$$
\mathrm{CI}=\frac{\lambda_{\max }-N}{N-1},
$$

Where $\lambda_{\max }$ is the maximum eigenvalue of $\boldsymbol{R}=\left(r_{i j}\right)_{M \times N}$; $N$ is the order of $\boldsymbol{R}=\left(r_{i j}\right)_{M \times N}$.

According to the AHP theory, if $N$ is known, the standard consistency index RI can be obtained as shown in Table 5 .

Calculate the consistency ratio $\mathrm{CR}$

$$
\mathrm{CR}=\frac{\mathrm{CI}}{\mathrm{RI}}
$$

If $\mathrm{CR}<0.10$, the judgment matrix is acceptable, if not, the judgment matrix should be modified appropriately by changing $r_{i j}$.

(4) The solution of the subjective weight. For the judgment matrix satisfying the consistency test, the
TABLE 5: The standard consistency index.

\begin{tabular}{lc}
\hline$N$ & $\mathrm{RI}$ \\
\hline 1 & 0 \\
2 & 0 \\
3 & 0.52 \\
4 & 0.89 \\
5 & 1.12 \\
6 & 1.24 \\
\hline
\end{tabular}

subjective weight of the unilateral kinematic ability can be obtained according to $\lambda_{\max }$. Then, we calculate the eigenvector of $\lambda_{\max }$, and take it as $\left(\omega_{1}, \cdots, \omega_{N}\right)^{T}$, which corresponds to the subjective weight of each unilateral kinematic ability $\omega_{1} \ldots$ $\omega_{N}$, respectively

Based on the above steps, the subjective weight of $\widehat{s}, \widehat{w}, \widehat{k}$ can be obtained, which is denoted as

$$
\omega_{\mathrm{CR}}=\left[\omega_{\mathrm{CR} \_1}, \omega_{\mathrm{CR} \_2}, \omega_{\mathrm{CR} \_3}\right] .
$$

Next, the entropy method is used to solve the objective weight of the unilateral kinematic ability. The configuration of the space manipulator with a joint-locked failure in the halt process can be expressed as $\boldsymbol{q}_{\lambda}=\left[\begin{array}{llll}\theta_{1} & \theta_{2} & \cdots & \theta_{n-1}\end{array}\right]$, where $\lambda(\lambda=1,2, \cdots K)$ represents the $\lambda$-th configuration and $K$ is the total number of configurations.

When the manipulator is in the $\lambda$-th configuration, the proportion of the $h$-th unilateral kinematic ability in the total value of the kinematic ability is $P_{\lambda \_h}$, which can be calculated by the following formula

$$
P_{\lambda \_h}=\frac{X_{\lambda \_h}}{\sum_{\lambda=1}^{K} X_{\lambda \_h}} \quad(h=1,2,3),
$$

where $X_{\lambda_{-} h}$ represents the value of the $h$-th unilateral kinematic ability when the manipulator is in the $\lambda$-th configuration.

And then the entropy value of the characterization of the $h$-th unilateral kinematic ability can be obtained as

$$
e_{h}=-\frac{\sum_{\lambda=1}^{K}\left(P_{\lambda \_h} \log \left(P_{\lambda \_h}\right)\right)}{\ln \left(\sum h\right)},
$$

where $0 \leq e_{h} \leq 1$.

The difference coefficient $g_{h}$ can be calculated as

$$
g_{h}=1-e_{h}
$$

The weight of each unilateral kinematic ability can be obtained as

$$
\omega_{\mathrm{CP} \_h}=\frac{g_{h}}{\sum_{h=1}^{3} g_{h}},
$$


Where $\omega_{\mathrm{CP} \_h}(h=1,2,3)$ represents the weight of $h$-th unilateral kinematic ability, which can be written as

$$
\boldsymbol{\omega}_{\mathrm{CP}}=\left[\omega_{\mathrm{CP}_{-} 1}, \omega_{\mathrm{CP}_{-} 2}, \omega_{\mathrm{CP}_{-} 3}\right] .
$$

According to Equation (12) and Equation (17), the final weight of each unilateral kinematic ability can be obtained as

$$
\begin{aligned}
\boldsymbol{\omega}= & \boldsymbol{\omega}_{\mathrm{CR}}+\boldsymbol{\omega}_{\mathrm{CP}}=\left[\omega_{\mathrm{CR} \_1}+\omega_{\mathrm{CP}_{-} 1}, \omega_{\mathrm{R} \_2}+\omega_{\mathrm{CP} \_2}, \omega_{\mathrm{CR} \_3}+\omega_{\mathrm{CP} \_3}\right] \\
& =\left[\omega_{C_{-} 1}, \omega_{C_{-} 2}, \omega_{C_{-} 3}\right] .
\end{aligned}
$$

According to the above analysis, the comprehensive characterization of the kinematic ability can be expressed as

$\mathrm{CP}\left(\boldsymbol{q}_{\lambda}\right)=\omega_{C_{-} 1} \times \widehat{s}\left(\boldsymbol{q}_{\lambda}\right)+\omega_{C_{-} 2} \times \widehat{k}\left(\boldsymbol{q}_{\lambda}\right)+\omega_{C_{-} 3} \times \widehat{w}\left(\boldsymbol{q}_{\lambda}\right) \quad(\lambda=1,2, \cdots, K)$,

where $\operatorname{CP}\left(\boldsymbol{q}_{\lambda}\right)$ is the comprehensive characterization of the kinematic ability when the manipulator is in the $\lambda$-th configuration.

At this point, the comprehensive characterization of the kinematic ability of the manipulator is completed.

3.2. The Construction and Solution of the HCOM. In this section, the HCOM is constructed based on the objective of maximizing the comprehensive characterization of the kinematic ability, and the optimal halt configuration is obtained by solving the HCOM with the Monte Carlo method.

According to Equation (1) and Equation (19), the HCOM can be expressed as

$$
\begin{aligned}
& \text { find } \boldsymbol{q}_{\text {best }}, \\
& \max \operatorname{CP}\left(\boldsymbol{q}_{\text {best }}\right) \text {, }
\end{aligned}
$$

where $\boldsymbol{q}_{\text {best }}$ is the optimal halt configuration.

Next, the HCOM is solved by utilizing the Monte Carlo method. The specific method is shown as

Step 1. For each joint $\tilde{J}_{i}$, the random numbers of each joint angle can be generated by using the random number method: $\boldsymbol{N}_{i}=\left[\begin{array}{llll}N_{i_{-} 1} & N_{i_{-} 2} & \cdots & N_{i_{-} K}\end{array}\right] \in \mathfrak{R}^{1 \times K}$, which are between 0 and 1 , and then the pseudorandom number of each joint angle can be obtained as

$$
\begin{aligned}
\widetilde{\boldsymbol{\theta}}_{i} & =\widetilde{\boldsymbol{\theta}}_{i \min }+\Delta \widetilde{\theta}_{i} \times \boldsymbol{N}_{i} \\
& =\left[\begin{array}{llll}
\widetilde{\theta}_{i \min }+\Delta \widetilde{\theta}_{i} \cdot N_{i_{-} 1} & \widetilde{\theta}_{i \min }+\Delta \widetilde{\theta}_{i} \cdot N_{i_{-} 2} & \cdots & \widetilde{\theta}_{i \min }+\Delta \widetilde{\theta}_{i} \cdot N_{i_{-} K}
\end{array}\right],
\end{aligned}
$$

where $\widetilde{\boldsymbol{\theta}}_{i \min }=\left[\begin{array}{llll}\widetilde{\theta}_{i \min } & \widetilde{\theta}_{i \min } & \cdots & \widetilde{\theta}_{i \min }\end{array}\right] \in \mathfrak{R}^{1 \times K} ; \Delta \widetilde{\theta}_{i}$ $=\widetilde{\theta}_{i \max }-\widetilde{\theta}_{i \min } ; i=1, \cdots, n-1$.

Step 2. The pseudorandom number of the joint angles of each joint can be expressed as

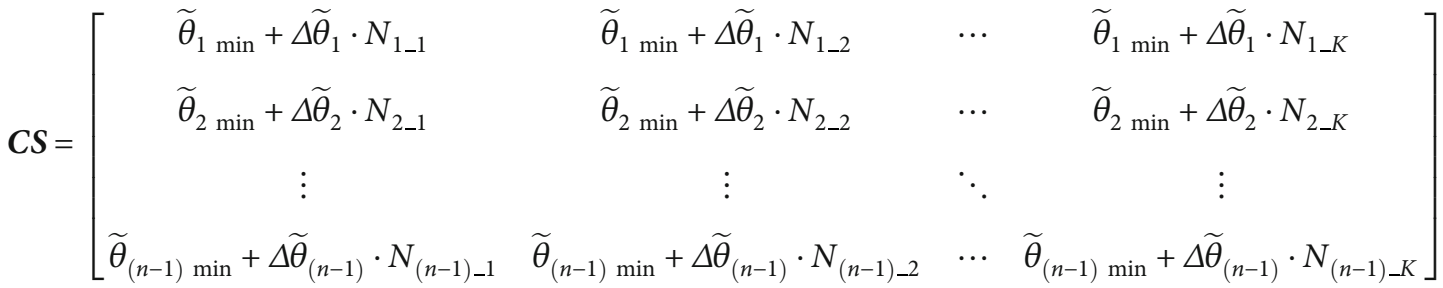

$$
\begin{aligned}
& =\left[\begin{array}{cccc}
\theta_{11} & \theta_{12} & \cdots & \theta_{1 K} \\
\theta_{21} & \theta_{22} & \cdots & \theta_{2 K} \\
\vdots & \vdots & \ddots & \vdots \\
\theta_{(n-1) 1} & \theta_{(n-1) 2} & \cdots & \theta_{(n-1) K}
\end{array}\right] \in \mathfrak{R}^{(n-1) \times K} .
\end{aligned}
$$

Each column corresponds to a certain configuration, and Equation (22) can be written as
And the configuration space of the manipulator can be obtained as

$$
\boldsymbol{C S}=\left[\begin{array}{llll}
\boldsymbol{q}_{1} & \boldsymbol{q}_{2} & \cdots & \boldsymbol{q}_{K}
\end{array}\right] \in \mathfrak{R}^{1 \times K} .
$$

$$
\boldsymbol{S}=\left\{\boldsymbol{q}_{1}, \boldsymbol{q}_{2}, \cdots, \boldsymbol{q}_{\lambda}, \cdots, \boldsymbol{q}_{K}\right\} \quad \lambda=1,2, \cdots, K
$$


Step 3. Select a configuration $\boldsymbol{q}_{\text {best }}=$ $\left[\begin{array}{llll}\theta_{1 \text { best }} & \theta_{2 \text { best }} & \cdots & \theta_{(n-1) \text { best }}\end{array}\right]$, which maximums $\operatorname{CP}\left(\boldsymbol{q}_{\text {best }}\right)$ in Equation (19).

So far, by solving the HCOM, the optimal halt configuration of the space manipulator is obtained.

\section{The Halt Motion Optimization}

To make the manipulator move continuously and stably in the halt process, the halt motion will be planned with a sixorder polynomial interpolation method which contains an optimal coefficient. By taking the limitation of the joint running parameters as constraint conditions, and the minimum disturbance torque of the base as the objective, the HMOM containing the optimal coefficient is constructed. And with the HMOM solved, the halt motion of the faulty manipulator is optimized.

4.1. The Motion Planning in Joint Space Based on Six-Order Polynomial Interpolation. According to the characteristics of the halt ( $S_{0}$ and $S_{f}$ are known), when $t_{f}$ is determined, the halt motion of the manipulator is a function interpolation problem that satisfies six constraints, which includes $\theta\left(t_{0}\right)$, $\theta\left(t_{f}\right), \dot{\theta}\left(t_{0}\right), \dot{\theta}\left(t_{f}\right), \ddot{\theta}\left(t_{0}\right)$, and $\ddot{\theta}\left(t_{f}\right)$. Therefore, the halt motion planning of the manipulator is carried out by using a six-order polynomial interpolation method, and the HMOP is described by introducing the optimization coefficient.

The joint variable of the manipulator can be described as

$$
\theta(t)=a_{0}+a_{1} t+a_{2} t^{2}+a_{3} t^{3}+a_{4} t^{4}+a_{5} t^{5}+a_{6} t^{6}
$$

where $a_{0} \sim a_{6}$ are the undetermined coefficients.

According to the characteristics of halt motion, Equation (25) can be written as

$$
\left\{\begin{array}{l}
\theta\left(t_{0}\right)=\theta_{0}=a_{0}, \\
\theta\left(t_{f}\right)=\theta_{f}=a_{0}+a_{1} t_{f}+a_{2} t_{f}^{2}+a_{3} t_{f}^{3}+a_{4} t_{f}^{4}+a_{5} t_{f}^{5}+a_{6} t_{f}^{6}, \\
\dot{\theta}\left(t_{0}\right)=\dot{\theta}_{0}=a_{1}, \\
\dot{\theta}\left(t_{f}\right)=a_{1}+2 a_{2} t_{f}+3 a_{3} t_{f}^{2}+4 a_{4} t_{f}^{3}+5 a_{5} t_{f}^{4}+6 a_{6} t_{f}^{5}, \\
\ddot{\theta}\left(t_{0}\right)=\ddot{\theta}_{0}=2 a_{2}, \\
\ddot{\theta}\left(t_{f}\right)=\ddot{\theta}_{f}=2 a_{2}+6 a_{3} t_{f}+12 a_{4} t_{f}^{2}+20 a_{5} t_{f}^{3}+30 a_{6} t_{f}^{4} .
\end{array}\right.
$$

Set the optimization coefficient $k$. According to Equation (26), let $a_{6}=k$ and we can obtain

$$
\left\{\begin{array}{l}
a_{0}=\theta_{0}, \\
a_{1}=\dot{\theta}_{0}, \\
a_{2}=\frac{\ddot{\theta}_{0}}{2}, \\
a_{3}=-t_{f}^{3} k+\frac{20\left(\theta_{f}-\theta_{0}\right)-t_{f}^{2}\left(3 \ddot{\theta}_{0}-\ddot{\theta}_{f}\right)-t_{f}\left(8 \dot{\theta}_{f}+12 \dot{\theta}_{0}\right)}{2 t_{f}^{3}}, \\
a_{4}=3 t_{f}^{2} k+\frac{t_{f}^{2}\left(3 \ddot{\theta}_{0}-2 \ddot{\theta}_{f}\right)+t_{f}\left(14 \dot{\theta}_{f}+16 \dot{\theta}_{0}\right)-30\left(\theta_{f}-\theta_{0}\right)}{2 t_{f}^{4}}, \\
a_{5}=-3 t_{f} k+\frac{\left(\ddot{\theta}_{f}-\ddot{\theta}_{0}\right) t_{f}^{2}-6\left(\dot{\theta}_{0}+\dot{\theta}_{f}\right) t_{f}+12\left(\theta_{f}-\theta_{0}\right)}{2 t_{f}^{5}}, \\
a_{6}=k .
\end{array}\right.
$$

According to Equation (27), the joint running parameters $\boldsymbol{V a r}=\left[\boldsymbol{q}_{t}, \dot{\boldsymbol{q}}_{t}, \ddot{\boldsymbol{q}}_{t}, \boldsymbol{\tau}_{t} \ldots\right]$ can be obtained at every moment in the halt process, which is the function with $k$ as the variable, so it can be written as $\operatorname{Var}=\operatorname{Var}(k)$. Therefore, the halt motion can be optimized by optimizing the coefficient.

4.2. The Construction and Solution of the HMOM. In this section, considering the characteristics of the base and the safety of the halt, we construct the HMOM, where the minimum disturbance torque of the base is taken as the objective and the limitation of the joint running parameters as the constraint condition.

According to the Newton-Euler dynamics equations, the resultant force $\boldsymbol{f}_{i}$ and moment $\boldsymbol{n}_{i}$ of each joint can be expressed as $\left[\begin{array}{ll}\boldsymbol{f}_{i} & \boldsymbol{n}_{i}\end{array}\right]^{T}=\left[\begin{array}{llllll}\boldsymbol{f}_{i x} & \boldsymbol{f}_{i y} & \boldsymbol{f}_{i z} & \boldsymbol{n}_{i x} & \boldsymbol{n}_{i y} & \boldsymbol{n}_{i z}\end{array}\right]^{T}$.

The inertia force and moment of inertia of each link can be obtained as

$$
\begin{aligned}
{ }^{I} \tilde{\boldsymbol{F}}_{i} & =\tilde{m}_{i}{ }_{i} \dot{\tilde{\boldsymbol{v}}}_{i}, \\
{ }^{I} \tilde{\boldsymbol{N}}_{i} & ={ }^{I} \boldsymbol{R}_{i} \tilde{\boldsymbol{I}}_{i}^{I} \dot{\tilde{\boldsymbol{\omega}}}_{i}+{ }^{I} \tilde{\boldsymbol{\omega}}_{i} \times\left({ }^{I} \boldsymbol{R}_{i} \tilde{\boldsymbol{I}}_{i}^{I} \tilde{\boldsymbol{\omega}}_{i}\right) .
\end{aligned}
$$

The relationship of the force and torque between the joints are

$$
\begin{aligned}
& { }^{I} \tilde{\boldsymbol{f}}_{i}={ }^{I} \tilde{\boldsymbol{F}}_{i}+{ }^{I} \tilde{\boldsymbol{f}}_{i+1}, \\
& { }^{I} \tilde{\boldsymbol{n}}_{i}={ }^{I} \tilde{\boldsymbol{N}}_{i}+\left({ }^{I} \boldsymbol{R}_{i}\left(\tilde{\boldsymbol{a}}_{i}+\tilde{\boldsymbol{b}}_{i}\right) \times{ }^{I} \tilde{\boldsymbol{f}}_{i+1}+{ }^{I} \tilde{\boldsymbol{n}}_{i+1}\right)+\left({ }^{I} \boldsymbol{R}_{i} \tilde{\boldsymbol{a}}_{i} \times{ }^{I} \tilde{\boldsymbol{F}}\right)_{i} .
\end{aligned}
$$

For the space manipulator, the 1-th joint is directly connected to the free-floating base. Therefore, according to the model diagram shown in Figure 5, the disturbance torque of the base $\tilde{\boldsymbol{n}}_{\mathrm{b}}$ can be calculated by $\left[\begin{array}{ll}{ }^{I} \tilde{\boldsymbol{f}}_{1} & { }^{I} \tilde{\boldsymbol{n}}_{1}\end{array}\right]^{T}$. 


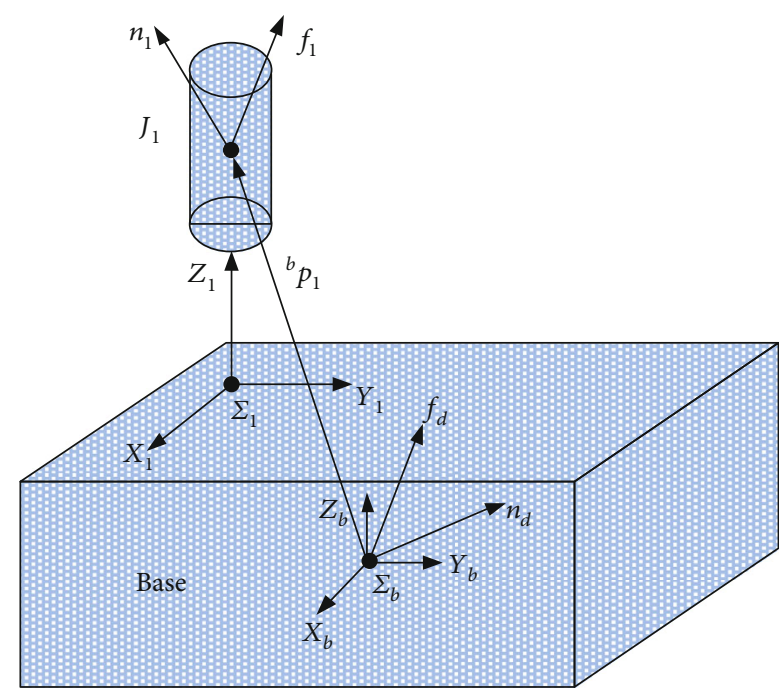

Figure 5: Solving model diagram of the disturbance torque.

According to the equivalence principle of space force/torque, the disturbance force and torque of the base can be expressed as

$$
\begin{aligned}
& { }^{I} \tilde{\boldsymbol{f}}_{d}={ }^{b} \boldsymbol{R}_{1}{ }^{I} \tilde{\boldsymbol{f}}_{1}, \\
& { }^{I} \tilde{\boldsymbol{n}}_{d}={ }^{b} \boldsymbol{R}_{1}{ }^{I} \tilde{\boldsymbol{n}}_{1}+{ }^{b} \boldsymbol{P}_{1} \times{ }^{I} \tilde{\boldsymbol{f}}_{1},
\end{aligned}
$$

where ${ }^{I} \tilde{\boldsymbol{f}}_{d}=\left[\begin{array}{lll}\tilde{\boldsymbol{f}}_{d x} & \tilde{\boldsymbol{f}}_{d y} & \tilde{\boldsymbol{f}}_{d z}\end{array}\right]^{T},{ }^{I} \tilde{\boldsymbol{n}}_{d}=\left[\begin{array}{lll}\tilde{\boldsymbol{n}}_{d x} & \tilde{\boldsymbol{n}}_{d y} & \tilde{\boldsymbol{n}}_{d z}\end{array}\right]^{T}$.

According to Equation (30), the disturbance torque of the base in the halt process can be written as

$$
\boldsymbol{\tau}_{b}(k)=\left[\begin{array}{lll}
\tilde{\boldsymbol{n}}_{d x} & \tilde{\boldsymbol{n}}_{d y} & \tilde{\boldsymbol{n}}_{d z}
\end{array}\right]^{T} .
$$

To reflect the overall change level of the disturbance torque during the whole halt process, the disturbance torque of the base is globalized as

$$
\bar{\tau}_{b}(k)=\frac{\int_{t_{0}}^{t_{f}}\left(\left\|\boldsymbol{\tau}_{b}\right\|\right) d t}{t_{f}-t_{0}}
$$

where $\left\|\boldsymbol{\tau}_{\mathrm{b}}\right\|$ represents the 2-norm of $\boldsymbol{\tau}_{b}$.

Considering the safety and stability of the halt, the joint running parameters need to be constrained within a range as follows

$\lim (k)=\left\{\boldsymbol{q}_{t}(k) \in\left[\boldsymbol{q}_{\min }, \boldsymbol{q}_{\max }\right], \dot{\boldsymbol{q}}_{t}(k) \in\left[\dot{\boldsymbol{q}}_{\text {min }}, \dot{\boldsymbol{q}}_{\text {max }}\right], \ddot{\boldsymbol{q}}_{t}(k) \in\left[\ddot{\boldsymbol{q}}_{\min }, \ddot{\boldsymbol{q}}_{\max }\right]\right\}$.

According to the above analysis, the HMOM can be expressed as

$$
\begin{aligned}
& \text { find } k \\
& \min \quad \bar{\tau}_{b}(k) \\
& \text { s.t. } \quad \lim (k) .
\end{aligned}
$$

So far, the construction of the HMOM has been completed, and the next step is to solve the HMOM.

A genetic algorithm is a method to search for the optimal solution by simulating the natural evolution process. It has a good ability to search for the optimal global solution and is widely used in solving optimization problems. The basic idea is to use the population search technology to take the population as a set of solutions and generate a new generation of population by applying a series of genetic operations to the current population, such as selection, crossover, and variation, and gradually optimize the population to the state containing the approximate optimal solution. We solve the HMOM by using a genetic algorithm, which can achieve the selection of the optimization coefficient $k$ :

Specific steps for solving the HMOM based on genetic algorithm are shown as follows

Step 1. Initialization: according to the accuracy of the solution and the solution space of $k$, the binary coding method is selected to encode the population, and the initial population in the coding space is obtained. Meanwhile, the current evolution algebra is set as $G=0$, and the maximum evolution algebra is set as $G_{\max }$.

Step 2. Individual evaluation: set the fitness function to calculate the fitness of $k$ in the population. The reciprocal of $\bar{\tau}_{b}(k)$ can be selected as the fitness function

$$
f=\left(\bar{\tau}_{b}(k)\right)^{-1} .
$$

According to the fitness function, the coefficient which meets $\max f$ in the current population is obtained.

Step 3. Genetic operator: selection, crossover, and variation.

(1) Select Operation. The superior coefficient $k$ in STEP 2 is passed to the next generation directly or by pairing crossover. In this paper, we use the elite mechanism for selection, that is, the coefficient corresponding to the highest fitness must be selected, and the probability of each coefficient being selected is proportional to the fitness value. Calculate the probability of each coefficient which is selected

$$
p_{d}=\frac{f\left(k_{d}\right)}{\sum_{d=1}^{\xi} f\left(k_{d}\right)}
$$

where $\xi$ is the number of individuals in the initial population.

(2) Crossover Operations. The crossover probability $p_{c}$ is calculated and the crossover operation between two 


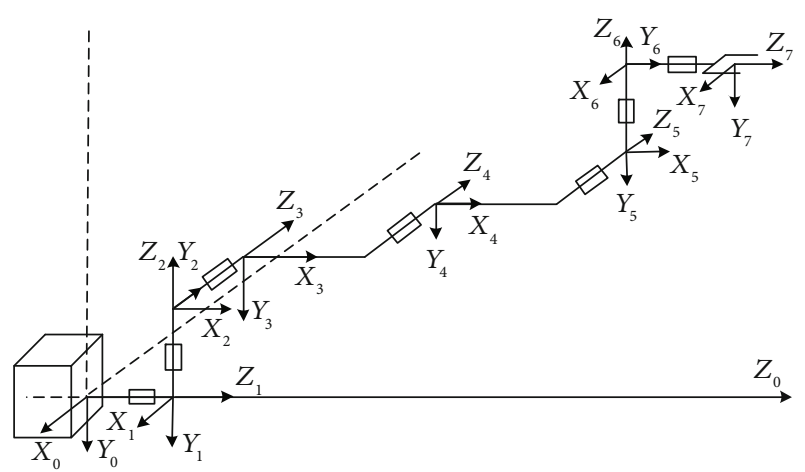

FIGURE 6: The kinematic model of the 7-DOF space manipulator.

TABLE 6: Initial DH parameters of the 7-DOF space manipulator.

\begin{tabular}{ccccc}
\hline$i$ & $\theta_{i}\left({ }^{\circ}\right)$ & $d_{i}(\mathrm{~m})$ & $a_{i}(\mathrm{~m})$ & $\alpha_{i}\left({ }^{\circ}\right)$ \\
\hline 0 & $\backslash$ & $\backslash$ & 0 & 0 \\
1 & 0 & 0.6 & 0 & 90 \\
2 & 90 & 0.5 & 0 & -90 \\
3 & 0 & 0.5 & 5 & 0 \\
4 & 0 & 0.5 & 5 & 0 \\
5 & 0 & 0.5 & 0 & 90 \\
6 & -90 & 0.5 & 0 & -90 \\
7 & 0 & 0.6 & $\backslash$ & $\backslash$ \\
\hline
\end{tabular}

individuals is performed according to the crossover probability

$$
p_{c}=\left\{\begin{array}{l}
\frac{g_{1}\left(f_{\max }-f\right)}{f_{\max }-f_{\text {avg }}}, f \geq f_{\text {avg }}, \\
g_{2}, f<f_{\text {avg }},
\end{array}\right.
$$

where $f_{\max }$ is the maximum fitness value, $f_{\text {avg }}$ is the average fitness value, $f$ is the larger fitness value of the two individuals, and $g_{1}$ and $g_{2}$ are constant.

(3) Mutation Operation. The mutation probability is calculated and the individual is calculated according to the mutation probability

$$
p_{m}=\left\{\begin{array}{l}
\frac{g_{3}\left(f_{\max }-f^{\prime}\right)}{f_{\max }-f_{\mathrm{avg}}}, f^{\prime} \geq f_{\mathrm{avg}}, \\
g_{4}, f^{\prime}<f_{\text {avg }},
\end{array}\right.
$$

where $f^{\prime}$ is the fitness value of the individual which is going to perform the mutation operation, and $g_{3}$ and $g_{4}$ are constant.

Step 4. Termination condition. If $G=G_{\max }$, the coefficient $k$ corresponding to the maximum fitness value is taken as the optimal solution; if not, repeat Step 2 to Step 4 .
TABLE 7: The dynamic parameters of the 7-DOF space manipulator.

\begin{tabular}{cccc}
\hline$i$ & $m_{i}(\mathrm{~kg})$ & {$\left[I_{x x i}, I_{y y i}, I_{z z i}\right]\left(\mathrm{kg} \cdot \mathrm{m}^{2}\right)$} & $\boldsymbol{P}_{c i}(\mathrm{~m})$ \\
\hline 1 & 42.5 & {$[0.89,0.05,0.89]$} & {$[0,0.25,0]^{T}$} \\
2 & 42.5 & {$[0.05,0.89,0.89]$} & {$[0,0.25,0]^{T}$} \\
3 & 70 & {$[0.088,145.83,145.83]$} & {$[2.5,0,0]^{T}$} \\
4 & 70 & {$[0.088,145.83,145.83]$} & {$[2.5,0,0]^{T}$} \\
5 & 42.5 & {$[0.89,0.89,0.05]$} & {$[0,0,-0.25]^{T}$} \\
6 & 42.5 & {$[0.89,0.89,0.05]$} & {$[0,0,-0.25]^{T}$} \\
7 & 42.5 & {$[1.28,1.28,0.05]$} & {$[0,0,-0.3]^{T}$} \\
\hline
\end{tabular}

Based on the above process, the solution of HMOM is completed, which can ensure the steady halt of the manipulator to the optimal halt configuration.

\section{Simulation}

In this paper, a 7-DOF space manipulator is used as the simulate object to verify the halt optimization method, which is shown in Figure 6. The DH and dynamic parameters of the space manipulator are shown in Tables 6 and 7, respectively.

5.1. The Halt Configuration Optimization. Firstly, the AHP method is used to solve the subjective weight of each unilateral kinematic ability, and the judgment matrix of Target-Criterion is constructed according to Table 4, which is shown as

$$
\boldsymbol{R}=\left[\begin{array}{ccc}
1 & 1 / 2 & 1 \\
2 & 1 & 2 \\
1 & 1 / 2 & 1
\end{array}\right]
$$

The eigenvalue of the judgment matrix is $\lambda=3$, and the eigenvector of $\lambda=3$ is $(1 / 4,1 / 2,1 / 4)^{T}$. The result of the consistency test is $\mathrm{CR}=0<0.10$, so the consistency of the judgment matrix is acceptable.

According to the eigenvector of $\lambda=3$, the subjective weights of $\widehat{s}, \widehat{w}$, and $\widehat{k}$ are $1 / 4,1 / 2$, and $1 / 4$, respectively. The comprehensive kinematic ability of the manipulator is simulated by combining with the traditional entropy method.

Suppose there is a locked failure on the 4-th joint, the initial halt configuration except for the locked joint is $\mathbf{q}_{0}=$

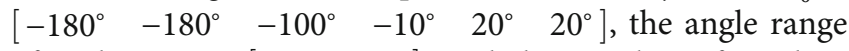
of each joint is $\left[-180^{\circ}, 180^{\circ}\right]$, and the number of random numbers is $K=36000$. According to Equation (19), it can be seen that the CP is a set of the 7-dimensional array, that is, the angle of six joints correspond to a $\mathrm{CP}$ value. Therefore, when the Monte Carlo method is used to solve the problem, the simulation results should be a 7 -dimensional space diagram. To show the conclusion more conveniently and intuitively, the 7-dimensional space diagram can be converted into six 2-dimensional planar graphs in the form of a section diagram. The simulation results are shown in Figure 7. 


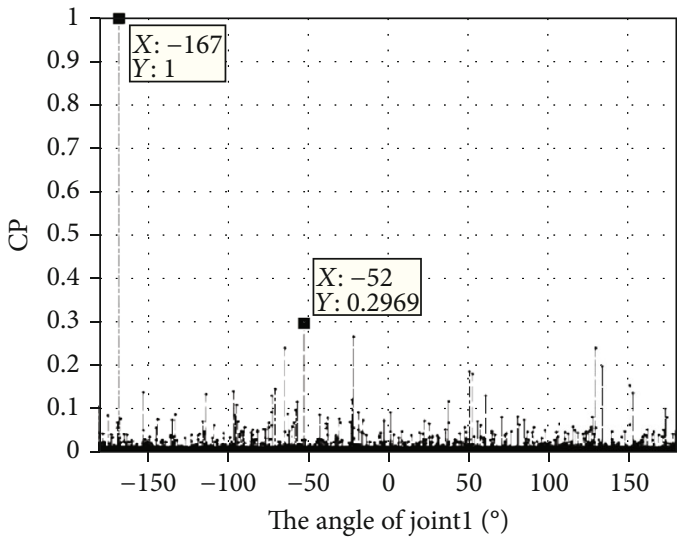

(a)

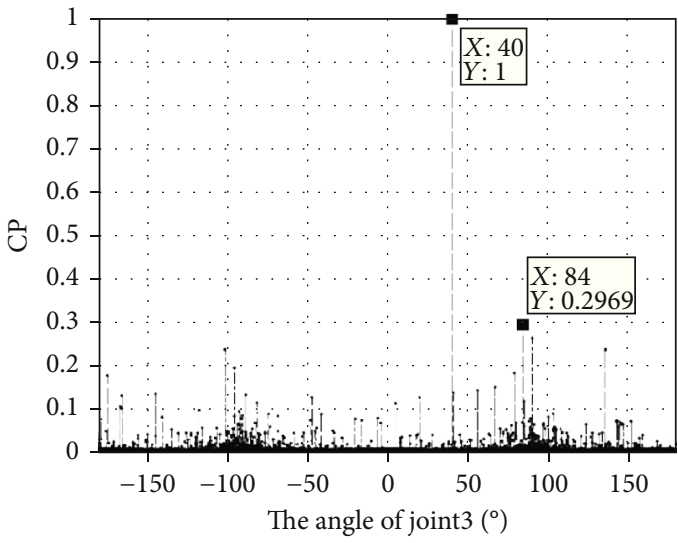

(c)

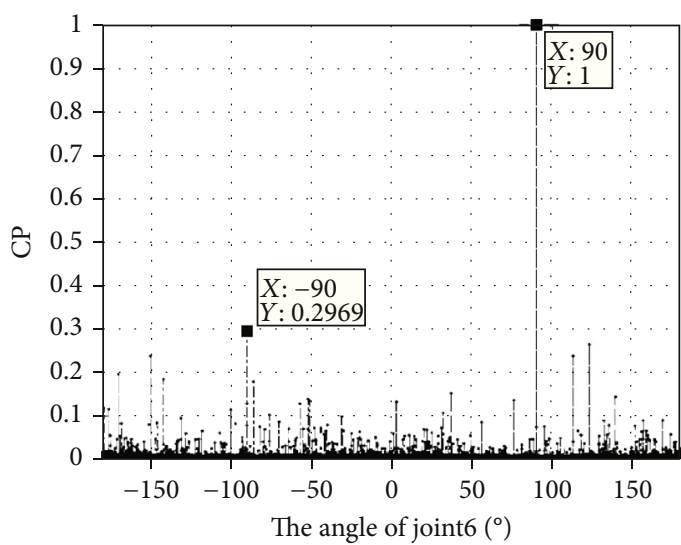

(e)

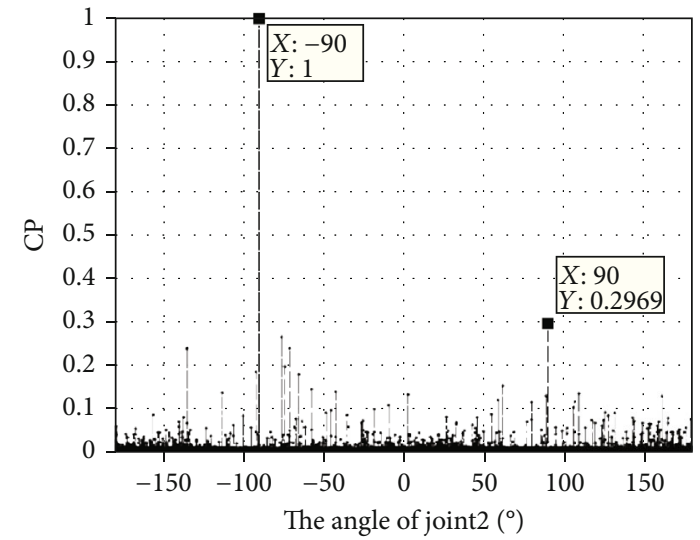

(b)

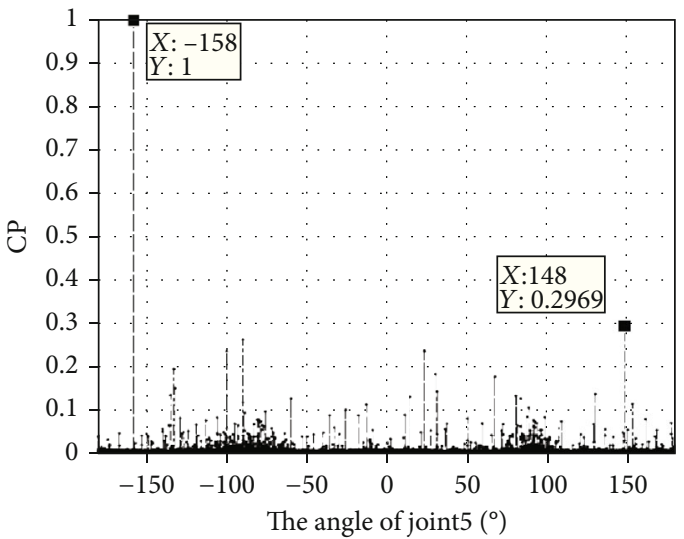

(d)

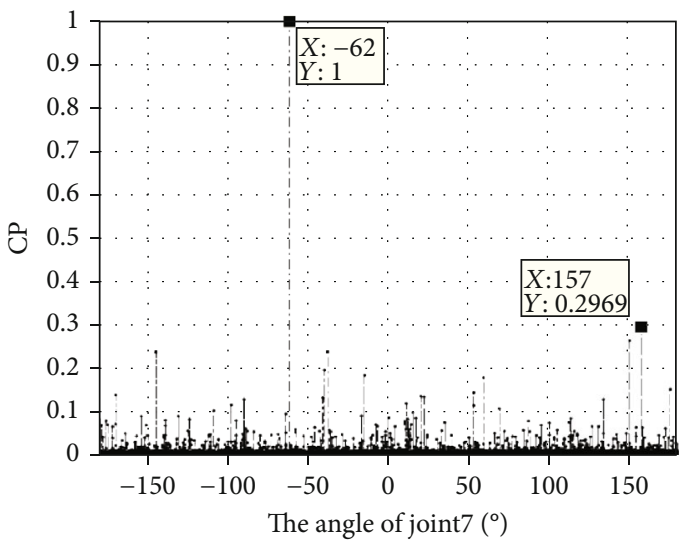

(f)

Figure 7: The simulation results of $\mathrm{CP}$ which are in the form of a 2-dimensional planar graph. (a) The angle of joint $1-\mathrm{CP}$. (b) The angle of joint $2-\mathrm{CP}$. (c) The angle of joint $3-\mathrm{CP}$. (d) The angle of joint $5-\mathrm{CP}$. (e) The angle of joint $6-\mathrm{CP}$. (f) The angle of joint 7-CP.

As we can see from Figure 7, the optimal halt configuration of the manipulator is $\mathbf{q}_{\text {best }}=\left[\begin{array}{lll}-167^{\circ} & -90^{\circ} 40^{\circ}-\end{array}\right.$ $158^{\circ} 90^{\circ}-62^{\circ}$. At the same time, it can be found that the suboptimal halt configuration is $\mathbf{q}_{\text {sub }}=\left[\begin{array}{ll}-52^{\circ} & 90^{\circ} 84^{\circ}\end{array}\right.$ $\left.148^{\circ}-90^{\circ} 157^{\circ}\right]$. The kinematic ability value of the manipulator in the suboptimal halt configuration is 0.2969. By the comparison, we can see that the value in the optimal halt configuration is much higher than that in the suboptimal halt configuration: $(1-0.2969) / 0.2969=236.8 \%$.

5.2. The Halt Motion Optimization. The optimal halt configuration of the manipulator with a joint-locked failure is $_{f}=\left[\begin{array}{llllll}-167^{\circ} & -90^{\circ} & 40^{\circ} & -158^{\circ} & 90^{\circ} & -62^{\circ}\end{array}\right]$, and the ini-

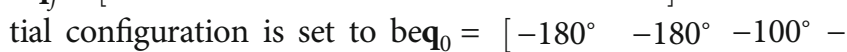




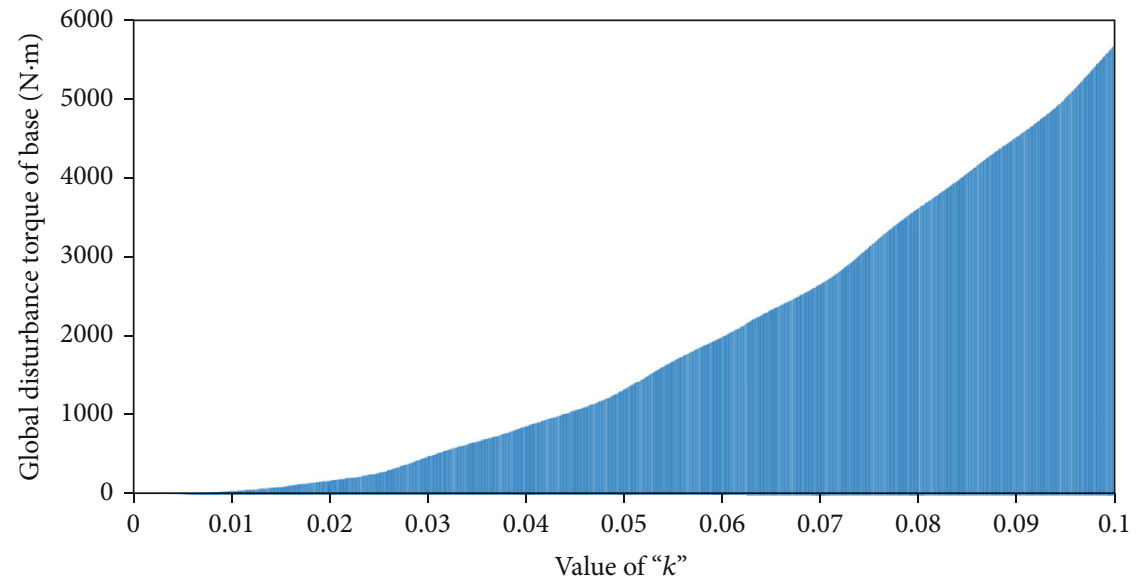

(a)



(b)

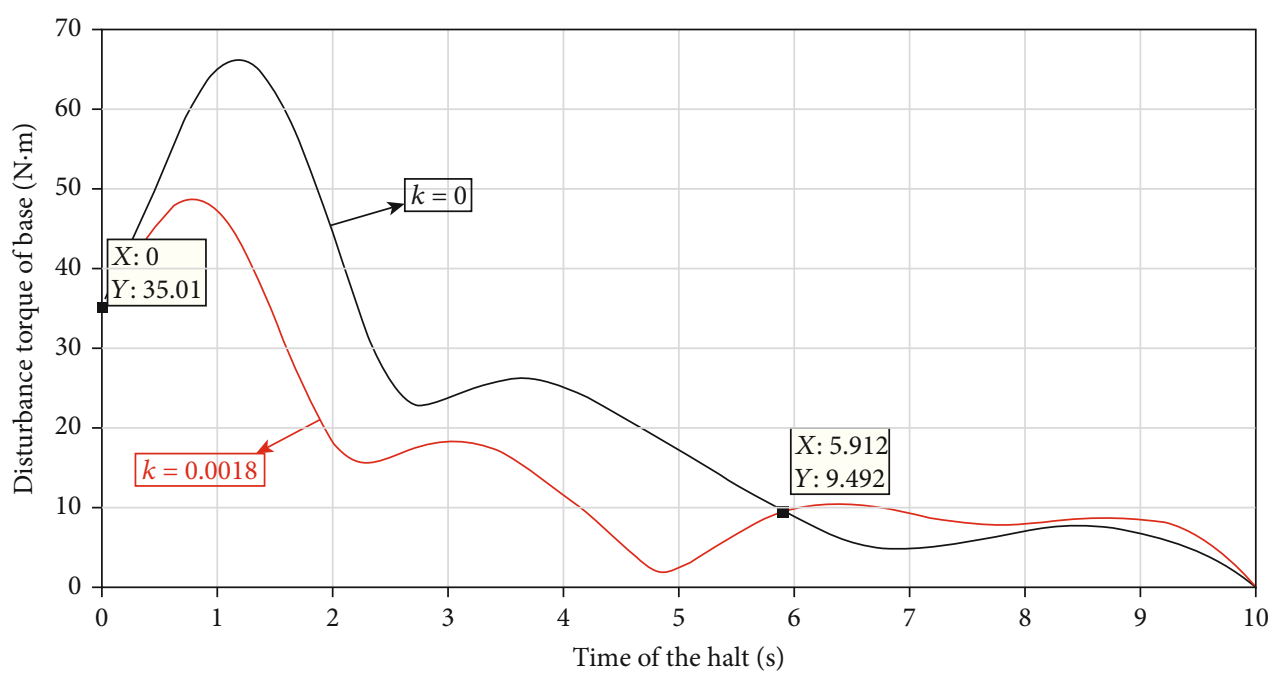

(c)

FIGURE 8: The variations of disturbance torque corresponding to different $k$. (a) The variations of disturbance torque when $k \in[0,0.1]$. (b) The variations of disturbance torque when $k \in[0,0.01]$. (c) The comparison of the disturbance torque between optimization and nonoptimization. 

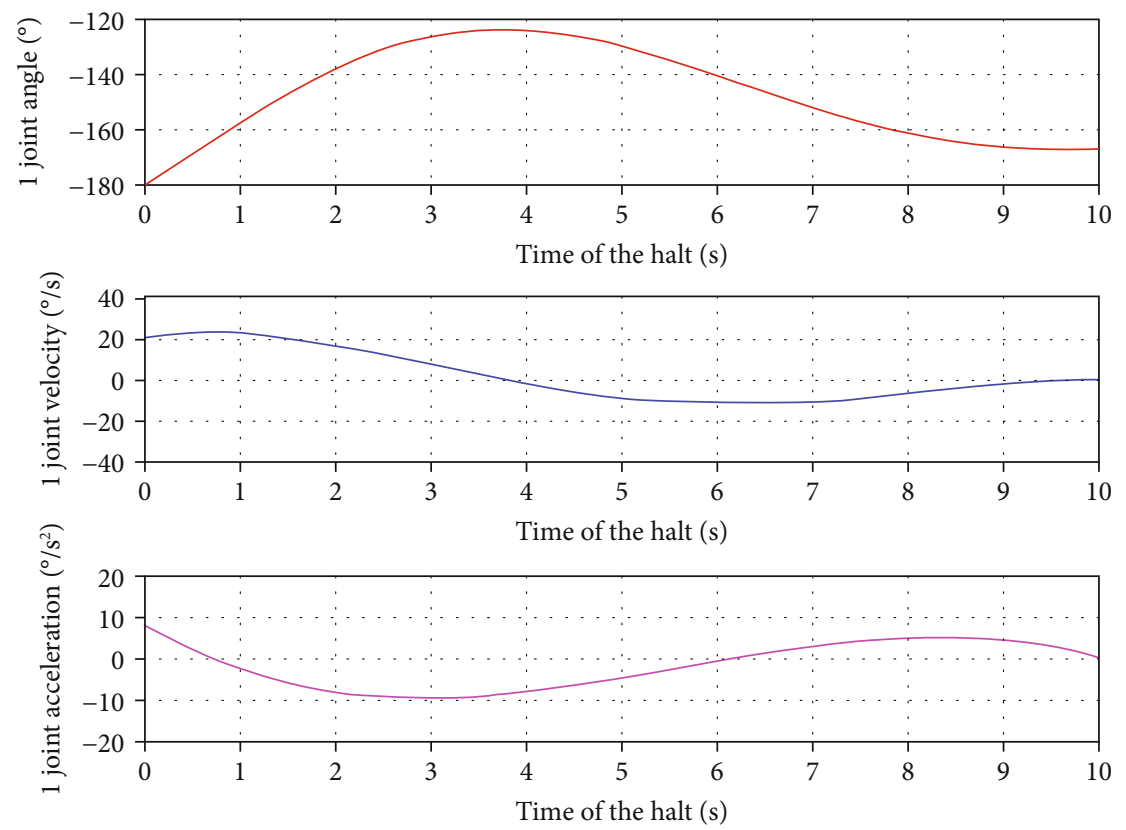

(a)


(b)

Figure 9: Continued. 

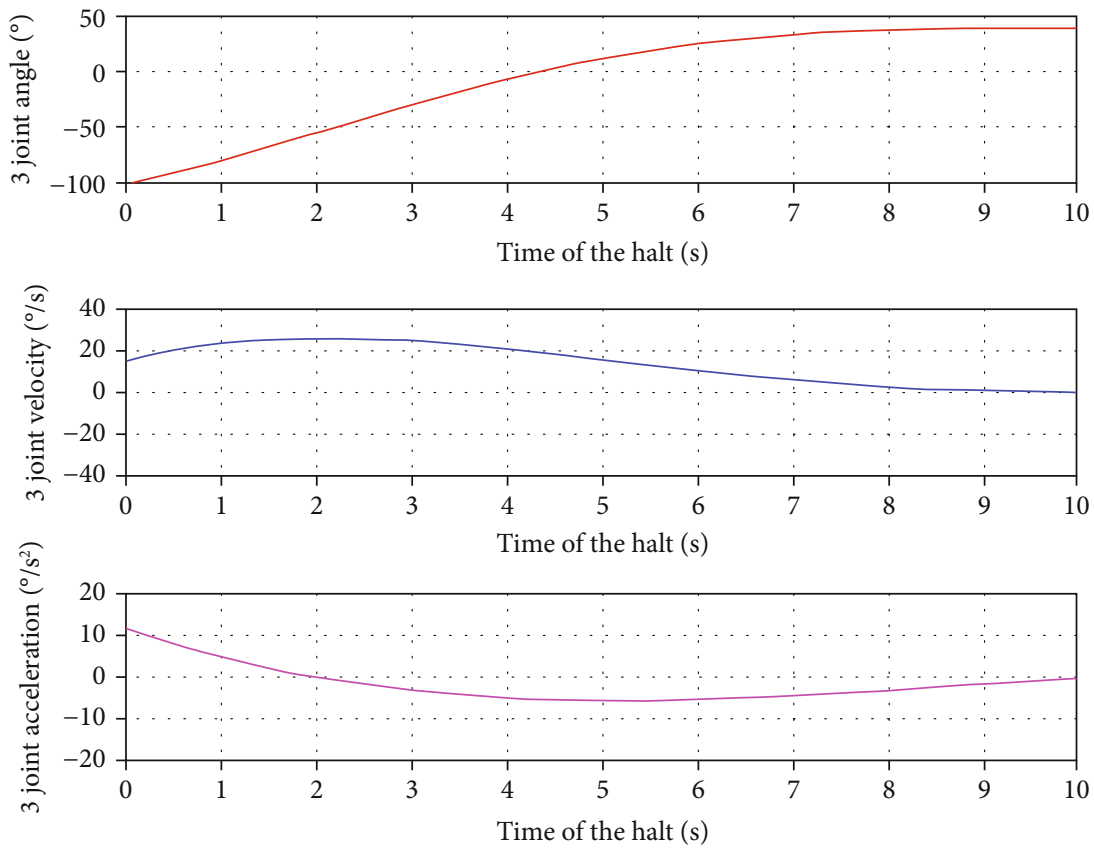

(c)
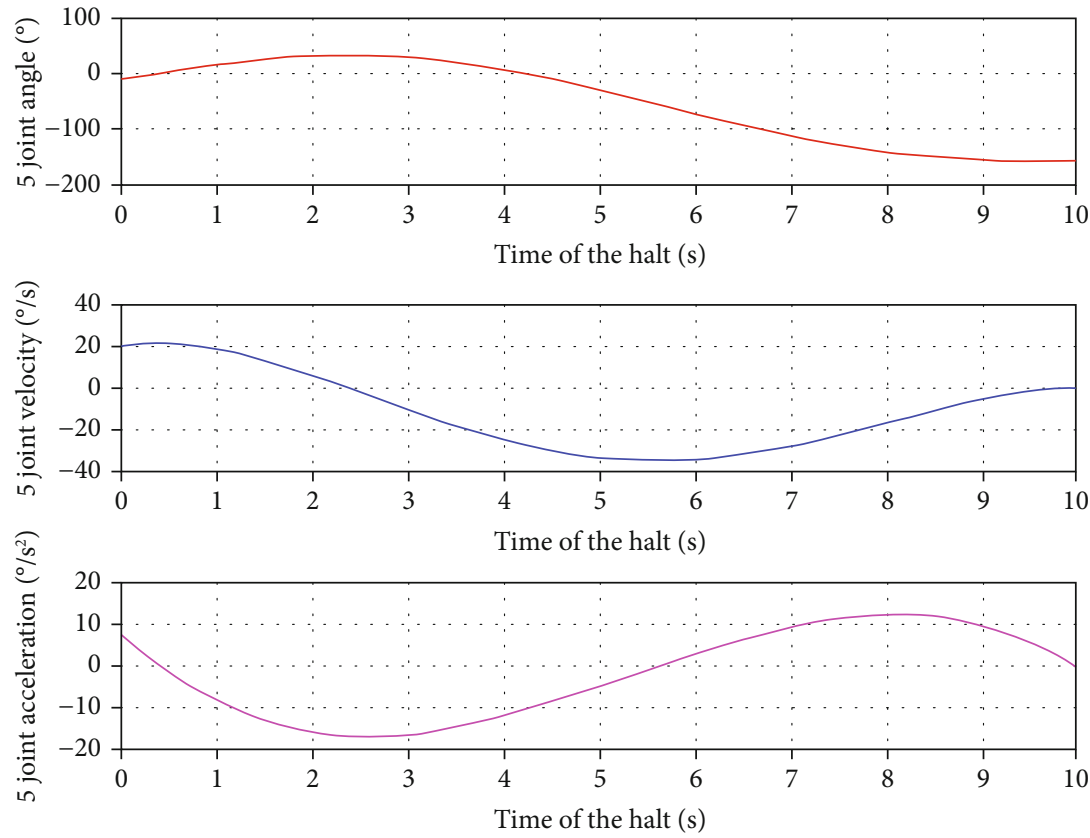

(d)

Figure 9: Continued. 



(e)
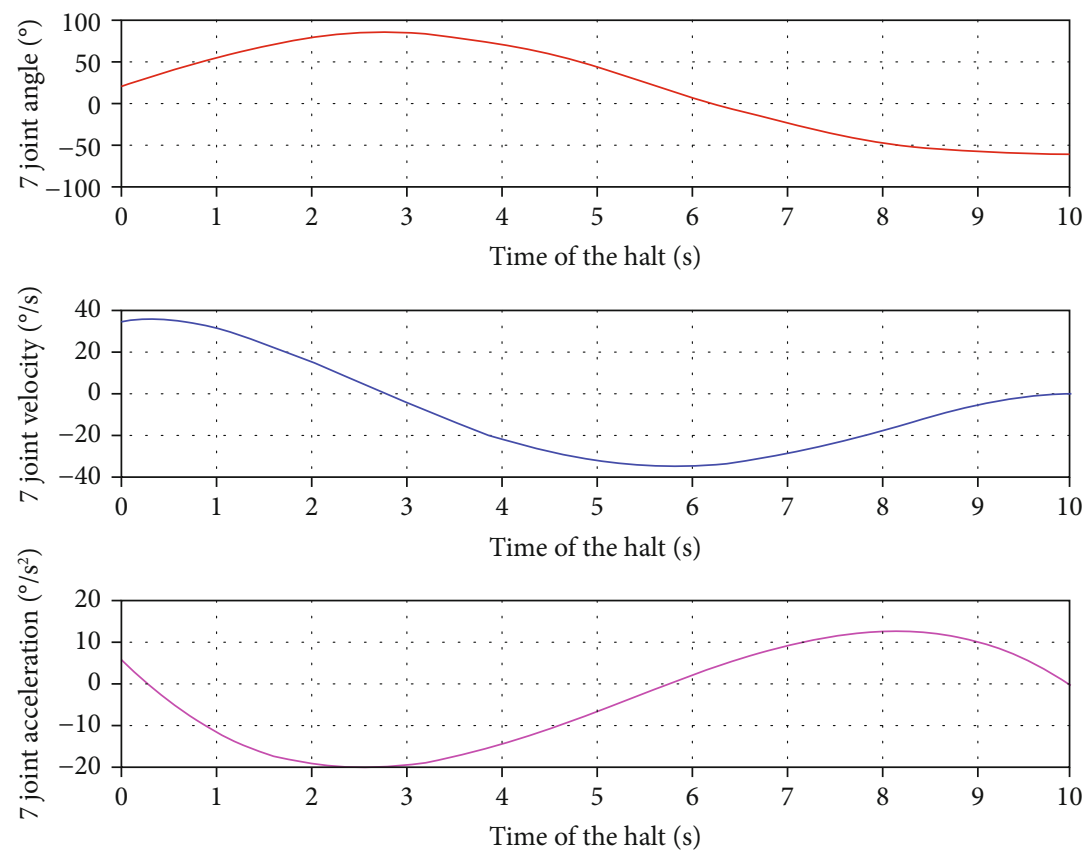

(f)

Figure 9: The variations of joint parameters. (a) The parameters of joint 1. (b) The parameters of joint 2. (c) The parameters of joint 3. (d) The parameters of joint 5 . (e) The parameters of joint 6 . (f) The parameters of joint 7.

$\left.10^{\circ} 20^{\circ} 20^{\circ}\right]$. Suppose the velocity of healthy joints is $\dot{\mathbf{q}}_{0}=\left[\begin{array}{llllll}20 & 30 & 15 & 25 & 40 & 35\end{array}\right]^{\circ} / \mathrm{s}$, the acceleration of healthy joints is $\ddot{\mathbf{q}}_{0}=\left[\begin{array}{llllll}8 & 10 & 12 & 10 & -8 & 6\end{array}\right]^{\circ} / \mathrm{s}^{2}$, the velocity constraint is $\left[\dot{\boldsymbol{q}}_{\min }, \dot{\boldsymbol{q}}_{\max }\right]=[-40,40](\%)$, the acceleration constraint is $\left[\ddot{\boldsymbol{q}}_{\min }, \ddot{\boldsymbol{q}}_{\max }\right]=[-20,20]\left(\% / \mathrm{s}^{2}\right)$, and the time of the entire halt process is $T=t_{f}-t_{0}=10 \mathrm{~s}$.
By solving the HMOM, the global variation of the base disturbance torque which changes with the coefficient $k$ in the halt process is shown in Figure 8. According to Figures $8(\mathrm{a})-8(\mathrm{c})$, it can be seen that when $k=0.0018$, the minimum global disturbance torque is $15.55 \mathrm{~N} \cdot \mathrm{m}$, and when $k=0$, the global disturbance torque is $22.25 \mathrm{~N} \cdot \mathrm{m}$. So the 
global disturbance torque of the base is reduced by $(22.25$ $-15.55) / 22.25=30.1 \%$ after the optimization.

When $k=0.0018$, the variations of joint parameters are shown in Figure 9. At this time, the global disturbance torque of the base is minimal, and the constraint of joint parameters is also satisfied.

\section{Conclusions}

In this paper, a halt optimization strategy for a space manipulator with a joint-locked failure is proposed. By describing the halt optimization problem, we creatively put forward the halt configuration optimization method. Considering the requirements of the operation task, the HCOM is constructed and solved to obtain the optimal halt configuration. According to the constraint of joint running parameters and the disturbance torque of the base, the HMOM is constructed, and by utilizing the joint space motion planning with the six-order polynomial interpolation method, we solve it to optimize the halt of the manipulator. Finally, the simulation is carried out to verify the effectiveness of the proposed strategy. The innovation of this paper mainly includes (1) the halt configuration optimization ensures that the manipulator in the halt configuration owns the best kinematic ability, and the kinematic ability in the optimal halt configure is $236.8 \%$ higher than that in the suboptimal halt configuration. (2) Each unilateral kinematic ability index is analyzed quantitatively, making the comprehensive representation of the kinematic ability more accurate. (3) The disturbance torque of the base is optimized during the halt process, and its global disturbance torque is reduced by $30 \%$ at least after the optimization.

\section{Data Availability}

The data used to support the findings of this study are available from the corresponding author upon request.

\section{Conflicts of Interest}

The authors declare that there is no conflict of interest regarding the publication of this paper.

\section{Acknowledgments}

All authors gratefully acknowledge the financial support by the National Natural Science Foundation of China under Grant 51975059, the Research Fund of the manned space engineering (No. 18051030101), and the Fundamental Research Funds for the Center Universities (Grant No. 2019PTB-012).

\section{References}

[1] M. Wang, J. Luo, J. Fang, and J. Yuan, "Optimal trajectory planning of free-floating space manipulator using differential evolution algorithm," Advances in Space Research, vol. 61, no. 6, pp. 1525-1536, 2018.

[2] Z. Chu, Y. Ma, Y. Hou, and F. Wang, "Inertial parameter identification using contact force information for an unknown object captured by a space manipulator," Acta Astronautica, vol. 131, pp. 69-82, 2017.

[3] F. Zhang and P. Huang, "Releasing dynamics and stability control of maneuverable tethered space net," IEEE/ASME Transactions on Mechatronics, vol. 22, no. 2, pp. 983-993, 2017.

[4] Z. Mu, T. Liu, W. Xu, Y. Lou, and B. Liang, "Dynamic feedforward control of spatial cable-driven hyper-redundant manipulators for on-orbit servicing," Robotica, vol. 37, no. 1, pp. 18$38,2019$.

[5] S. Ulrich, J. Z. Sasiadek, and I. Barkana, "Nonlinear adaptive output feedback control of flexible-joint space manipulators with joint stiffness uncertainties," Journal of Guidance, Control, and Dynamics, vol. 37, no. 6, pp. 1961-1975, 2014.

[6] J. D. English and A. A. Maciejewski, "Fault tolerance for kinematically redundant manipulators: anticipating free-swinging joint failures," IEEE Transactions on Robotics and Automation, vol. 14, no. 4, pp. 566-575, 1998.

[7] G. Chen, W. Guo, Q. X. Jia, and X. Wang, "Faulty tolerant path planning method for manipulator with single joint failure based on kinematics model reconstruction," Control and Decision, vol. 33, no. 8, pp. 1436-1442, 2018.

[8] Y. She, W. Xu, H. Su, B. Liang, and H. Shi, "Fault-tolerant analysis and control of SSRMS-type manipulators with single-joint failure," Acta Astronautica, vol. 120, pp. 270-286, 2016.

[9] R. C. Hoover, R. G. Roberts, A. A. Maciejewski, P. S. Naik, and K. M. Ben-Gharbia, "Designing a failure-tolerant workspace for kinematically redundant robots," IEEE Transactions on Automation Science and Engineering, vol. 12, no. 4, pp. 1421-1432, 2015.

[10] G. Chen, T. Li, Q. Jia, and H. Sun, "Reducing the joint parameter jump during the operation of the force/position Faulttolerant of end-effector for space manipulator," Journal of Mechanical Engineering, vol. 53, no. 11, pp. 81-89, 2017.

[11] D. N. Nenchev and K. Yoshida, "Impact analysis and postimpact motion control issues of a free-floating space robot subject to a force impulse," IEEE Transactions on Robotics and Automation, vol. 15, no. 3, pp. 548-557, 1999.

[12] Q. Jia, T. Li, G. Chen, H. Sun, and J. Zhang, "Trajectory optimization for velocity jumps reduction considering the unexpectedness characteristics of space manipulator joint-locked failure," International Journal of Aerospace Engineering, vol. 2016, Article ID 7819540, 14 pages, 2016.

[13] G. Chen, B. Yuan, Q. Jia, Y. Fu, and J. Tan, "Trajectory optimization for inhibiting the joint parameter jump of a space manipulator with a load-carrying task," Mechanism and Machine Theory, vol. 140, pp. 59-82, 2019.

[14] G. Chen, T. Li, Q. X. Jia, H. X. Sun, and J. Zhang, "Reducing the joint parameter jump during the operation of the force/position faulty-tolerant of end-effector for space manipulator," Journal of Mechanical Engineering, vol. 53, no. 11, pp. 81-89, 2017.

[15] H. Abdi and S. Nahavandi, "Joint velocity redistribution for fault tolerant manipulators," in 2010 IEEE Conference on Robotics, Automation and Mechatronics, pp. 492-497, Singapore, Singapore, June 2010.

[16] Y. Kamata, A. Ming, and M. Shimojo, "Motion control of a manipulator with mechanical joint stops," in 2007 IEEE International Conference on Robotics and Biomimetics (ROBIO), pp. 1451-1456, Sanya, China, December 2008.

[17] M. Dupac and S. Noroozi, "Dynamic modeling and simulation of a rotating single link flexible robotic manipulator subject to 
quick stops," Journal of Mechanical Engineering, vol. 60, no. 78, pp. 475-482, 2014.

[18] S. Nozawa, E. Kuroiwa, K. Kojima et al., "Multi-layered realtime controllers for humanoid's manipulation and locomotion tasks with emergency stop," in 2015 IEEE-RAS 15th International Conference on Humanoid Robots (Humanoids), pp. 381-388, Seoul, South Korea, November 2015.

[19] K. Sharma, S. Haddadin, S. Minning et al., "Evaluation of human safety in the DLR Robotic Motion Simulator using a crash test dummy," in 2013 IEEE International Conference on Robotics and Automation, pp. 205-212, Karlsruhe, Germany, May 2013.

[20] C. C. Liang, X. D. Zhang, Z. X. Tang, and X. Liu, "Suppression of velocity mutation caused by space manipulator joint failure," Journal of Astronautics, vol. 37, no. 1, pp. 48-54, 2016.

[21] C. Xu, A. Ming, and M. Shimojo, "Optimal trajectory generation for manipulator with strong nonlinear constraints and multiple boundary conditions," in 2004 IEEE International Conference on Robotics and Biomimetics, pp. 192-197, Shenyang, China, August 2005.

[22] Y. H. Gao, L. Hao, and Y. P. Li, "The control system of virtual simulation and speed optimization on assembly manipulator," Advanced Materials Research, vol. 1049-1050, pp. 934-938, 2014.

[23] V. K. Dalla and P. Pathak, "Power-optimized motion planning of reconfigured redundant space robot," Journal of Systems and Control Engineering, vol. 233, no. 8, pp. 1030-1044, 2018.

[24] F. Chen, M. Selvaggio, and D. G. Caldwell, "Dexterous grasping by manipulability selection for mobile manipulator with visual guidance," IEEE Transactions on Industrial Informatics, vol. 15, no. 2, pp. 1202-1210, 2019.

[25] X. Zhao, Z. Xie, H. Yang, and J. Liu, "Minimum base disturbance control of free-floating space robot during visual servoing precapturing process," Robotica, vol. 38, no. 4, pp. 652-668, 2020.

[26] W.-F. Xu, X. Q. Wang, Q. Xue, and B. Liang, "Study on trajectory planning of dual-arm space robot keeping the base stabilized," Acta Automatica Sinica, vol. 39, no. 1, pp. 69-80, 2013.

[27] Y. Umetani and K. Yoshida, "Workspace and manipulability analysis of space manipulator," Transactions of the Society of Instrument and Control Engineers, vol. 26, no. 2, pp. 188195, 2009.

[28] W. Xu, D. Meng, H. Liu, X. Wang, and B. Liang, "Singularityfree trajectory planning of free-floating multiarm space robots for keeping the base inertially stabilized," IEEE Transactions on Systems, Man, and Cybernetics: Systems, vol. 49, no. 12, pp. 1-14, 2019.

[29] X. Chen and S. Qin, "Motion planning for dual-arm space robot towards capturing target satellite and keeping the base inertially fixed," IEEE Access, vol. 6, pp. 26292-26306, 2018.

[30] X. Huang, Y. Jia, S. Xu, and S. Lu, “Trajectory planning of a space manipulator with constant zero-disturbance to base attitude," Journal of Beijing University of Aeronautics and Astronautics, vol. 43, no. 3, pp. 488-496, 2017.

[31] F. Feng, L. Tang, J. Xu, H. Liu, and Y. W. Liu, "A review of the end-effector of large space manipulator with capabilities of misalignment tolerance and soft capture," Science China Technological Sciences, vol. 59, no. 11, pp. 1621-1638, 2016.

[32] Y. Han and X. B. Yan, "Project evaluation model based on group decision-making vector optimization of AHP algorithm," Metallurgical and Mining Industry, vol. 7, no. 8, pp. 144-150, 2015. 